\title{
A ZERO DENSITY RESULT FOR THE RIEMANN ZETA FUNCTION
}

\author{
HABIBA KADIRI
}

\begin{abstract}
In this article, we prove an explicit bound for $N(\sigma, T)$, the number of zeros of the Riemann zeta function satisfying $\mathfrak{R e} s \geq \sigma$ and $0 \leq \mathfrak{I m} s \leq T$. This result provides a significant improvement to Rosser's bound for $N(T)$ when used for estimating prime counting functions.
\end{abstract}

\section{INTRODUCTION}

In recent years, it has become apparent that explicit results concerning prime numbers are required to solve important problems in number theory. In particular, the impressive works of Ramaré [18], Tao [30, and Helfgott [13] related to Goldbach's conjecture highlight the need of better explicit bounds for finite sums over primes. For instance, they make use of [4], [21], [22], 24], 25], 26], 28]. Moreover articles of Rosser and Schoenfeld ([24], [25], [26], 27], 28]), Dusart ([5], 6], [7], 8]), and Ramaré and Rumely [23] are extensively used in a wide range of fields including Diophantine approximation, cryptography, and computer science. These results on primes rely heavily on explicit estimates of sums over the non-trivial zeros of the Riemann zeta function. More precisely, they rely on three key ingredients: a numerical verification of the Riemann Hypothesis ( $\mathrm{RH})$, an explicit zerofree region, and explicit bounds for the number of zeros in the critical strip up to a fixed height $T$.

In 1986, van de Lune et al. [34] established that $\mathrm{RH}$ had been verified for all zeros $\varrho$ verifying $|\mathfrak{I m} \varrho| \leq H_{0}$ with $H_{0}=545439823$. In 2011, Platt [15] [16] proved that $H_{0}=30610046000$ is admissible. Previously, Wedeniwski [35] in 2001 and Gourdon [11] in 2004 had announced higher values for $H_{0}$. As Platt's computations are more rigourous (he employs interval arithmetic), we decide to use his value throughout this article:

$$
H_{0}=3.061 \cdot 10^{10} .
$$

For the latest explicit results about zero-free regions for the Riemann zeta function, we refer the reader to [14] and [10].

2010 Mathematics Subject Classification. Primary 11M06, 11M26; Secondary 11Y35.

Key words and phrases. Riemann zeta function, zero density, explicit results. 
Let $\sigma \geq 0.55$. We consider $N(\sigma, T)$, the number of zeros of the Riemann zeta function in the region $\sigma \leq \mathfrak{R e} s \leq 1$ and $0 \leq \mathfrak{I m} s \leq T$. Trivially we have that $N(\sigma, T)=0$ for all $T \leq H_{0}$. We prove here an explicit bound for $N(\sigma, T)$ valid in the range $T \geq H_{0}$.

Theorem 1.1. Let $\sigma \geq 0.55$ and $T \geq H_{0}$. Let $\sigma_{0}$ and $H$ such that $0.5208<$ $\sigma_{0}<0.9723, \sigma_{0}<\sigma$, and $10^{3} \leq H \leq H_{0}$. Then there exist $b_{1}, b_{2}, b_{3}$, positive constants depending on $\sigma, \sigma_{0}, H$, such that:

$$
N(\sigma, T) \leq b_{1}(T-H)+b_{2} \log (T H)+b_{3} .
$$

The $b_{i}$ 's are defined in (6.3).

We rewrite this as $N(\sigma, T) \leq c_{1} T+c_{2} \log T+c_{3}$, for $T \geq H_{0}$. Numerical values of the $b_{i}$ 's and $c_{i}$ 's are recorded at the end of this article in Table 1 , For example, for $\sigma \geq 17 / 20$ and $T \geq H_{0}$, we have

$$
N(\sigma, T) \leq 0.5561 T+0.7586 \log T-268658 .
$$

Let $N(T)$ be the number of non-trivial zeros $\varrho$ with imaginary part $0 \leq$ $\mathfrak{I m} \varrho \leq T$. We recall that Rosser [24] proved

$$
\left|N(T)-\frac{T}{2 \pi} \log \frac{T}{2 \pi e}-\frac{7}{8}\right| \leq a \log T+b \log \log T+c,
$$

with $a=0.137, b=0.443, c=1.588$. Note that Rosser's result got recently improved by Trudgian [33, Corollary 1] with $a=0.111, b=0.275, c=$ 2.450. A trivial bound for $N(\sigma, T)$ follows from the inequalities $N(\sigma, T) \leq$ $\frac{1}{2} N(T)$ and (1.1):

$$
N(\sigma, T) \leq \frac{T}{4 \pi} \log \left(\frac{T}{2 \pi e}\right)(1+o(1)) .
$$

Note that when $T$ is asymptotically large, then a factor of $\log T$ is saved. Moreover, we have $c_{1} \sim \frac{\log \zeta\left(2 \sigma_{0}\right)}{4 \pi\left(\sigma-\sigma_{0}\right)}$ where $\sigma_{0}$ is a parameter which value can be chosen to make $c_{1}$ as small as possible. Another feature of Theorem 1.1 is the factor $T-H$ : when $T$ is near $H_{0}$, we choose $H$ to be close to $H_{0}$ so as to make $N(\sigma, T)$ of size $\log H_{0}$. This saves a factor of size $H_{0}$. As an example, for $\sigma \geq 17 / 20$ and $T=H_{0}+1$, we choose $H=H_{0}-1$ and $\sigma_{0}$ as in Table 1 and obtain $N\left(\sigma, H_{0}+1\right) \leq 156$ while (1.1) gives $5.2 \cdot 10^{10}$ (with either Rosser's or Trudgian's values).

The key motivation for establishing Theorem 1.1 is to use it in place of (1.1) and thus to provide improved explicit bounds for Chebyshev's prime counting functions. We prove in [9] that, for all $x \geq e^{b}$,

$$
|\psi(x)-x| \leq \epsilon_{b} x
$$


where $b$ is a fixed positive constant, and $\epsilon_{b}$ is an effective positive constant. For example, for $x \geq e^{50}$ we obtain $\epsilon_{50}=9.461 \cdot 10^{-10}$ while Dusart [7, Theorem 2] obtained $0.905 \cdot 10^{-7}$.

Despite a very rich history of asymptotic results, there were almost no explicit bounds for $N(\sigma, T)$. Ramaré proved in an unpublished manuscript [19] that, for $T \geq 2000, Q \geq 10$, and $T \geq Q$,

$$
\sum_{q \leq Q} \sum_{\chi \bmod { }^{*} q} N(\sigma, T, \chi) \leq 157\left(Q^{5} T^{3}\right)^{1-\sigma} \log ^{4-\sigma}\left(Q^{2} T\right)+6 Q^{2} \log ^{2}\left(Q^{2} T\right),
$$

where $\sum_{\chi \text { mod }{ }^{*} q}$ denotes the sum over primitive Dirichlet characters $\chi$ to the modulus $q$, and $N(\sigma, T, \chi)$ counts the number of zeros $\varrho$ of the Dirichlet $L$-function $L(s, \chi)$ satisfying $\sigma<\mathfrak{R e} \varrho<1$ and $0<\mathfrak{I m} \varrho<T$. Taking $Q=10$ and restricting the left sum to $q=1$, it follows that

$$
N(\sigma, T) \leq 157\left(100000 T^{3}\right)^{1-\sigma} \log ^{4-\sigma}(100 T)+600 \log ^{2}(100 T) .
$$

Our main theorem improves Ramaré's result for certain values of $\sigma$ and $T$ : he obtains $N\left(17 / 20,10 \cdot H_{0}\right) \leq 2.675 \cdot 10^{12}$ while we have $N\left(17 / 20,10 \cdot H_{0}\right) \leq$ $3.404 \cdot 10^{10}$. In 2010, Cheng [3] obtained the weaker result:

$$
N(\sigma, T) \leq 453472.54 T^{8 / 3(1-\sigma)}(\log T)^{5}
$$

for all $\sigma \geq 5 / 8$ and $T \geq \exp (\exp (18)) \simeq 10^{28515762}$. His method is based on Ford's [10] effective version of Korobov-Vinogradov's bound for the Riemann zeta function. He applied (1.4) to deduce explicit results on primes between consecutive cubes. Note that Cheng's result is not valid in the region $T \leq$ $\exp (\exp (18))$ while most applications require bounds for $T$ as small as $H_{0}$.

In order to prove Theorem 1.1 we establish two intermediate theorems about $\zeta(s)$ in the critical strip: an effective version of a Dirichlet polynomial approximation, and an explicit estimate for the second moment.

Theorem 1.2. Let $t_{0}>0, s=\sigma+i t$ with $\sigma \geq 1 / 2, t \geq t_{0}$ and $c>\frac{1}{2 \pi}$. Then

$$
\zeta(s)=\sum_{1 \leq n<c t} \frac{1}{n^{s}}+R(s)
$$

with $|R(s)| \leq C(\sigma, c) t^{-\sigma}$, and

$$
C(\sigma, c)=\left(c+\frac{1}{2}+\frac{3 \sqrt{1+1 / t_{0}^{2}}}{2 \pi}\left(\frac{\zeta(2)}{2 \pi c}+1+\frac{1}{2 \pi c-1}\right)\right) c^{-\sigma} .
$$

We apply the theorem for $c=1$ and for $t_{0}$ the height of the first zero of zeta. 
Corollary 1.3. Let $\sigma \geq 1 / 2$ and $t \geq 14.1347$. Then

$$
\left|\zeta(s)-\sum_{1 \leq n<t} \frac{1}{n^{s}}\right| \leq c_{0} t^{-\sigma}, \quad \text { where } c_{0}=2.1946 .
$$

This is to compare to Proposition 1 of Cheng [2] who obtained 5.505 instead of $2.1946 t^{-\sigma}$. When $\sigma \geq 1 / 2$ and $0 \leq t \leq 15$, a Mathematica computation gives us that $\left|\zeta(s)-\sum_{1 \leq n<t} n^{-s}\right| \leq 43 t^{-\sigma}$.

Theorem 1.4. Let $0.5208<\sigma_{0}<0.9723$ and $10^{3} \leq H \leq H_{0}$. We define

$$
\begin{aligned}
\epsilon_{1}\left(\sigma_{0}, H\right)= & \frac{4 H_{0}}{H_{0}-H}\left(\frac{\left(\log H_{0}\right) H_{0}^{1-2 \sigma_{0}}}{2\left(1-\sigma_{0}\right)}-\frac{\left(2 \sigma_{0}-1\right) \log H_{0}}{2\left(1-\sigma_{0}\right) H_{0}}\right. \\
& +\frac{\max \left(0, \frac{1-3 \sigma_{0}+3 \sigma_{0}^{2}}{2\left(1-\sigma_{0}\right)^{2}}-\frac{\zeta\left(2 \sigma_{0}\right)}{2}\right)}{H_{0}}+\frac{\left(2-\sigma_{0}\right) H_{0}^{1-2 \sigma_{0}}}{2\left(1-\sigma_{0}\right)^{2}} \\
& \left.-\frac{\sigma_{0} H_{0}^{-\sigma_{0}}}{\left(1-\sigma_{0}\right)^{2}}+\frac{H_{0}^{-2 \sigma_{0}}}{2\left(2 \sigma_{0}-1\right)}+\frac{H_{0}^{-2 \sigma_{0}-1}}{2}\right), \\
\epsilon_{2}\left(\sigma_{0}, H\right)= & \frac{c_{0}^{2}}{2 \sigma_{0}-1} \frac{H^{-\left(2 \sigma_{0}-1\right)}-H_{0}^{-\left(2 \sigma_{0}-1\right)}}{H_{0}-H}, \\
\epsilon_{3}\left(\sigma_{0}, H\right)= & 2 \sqrt{\epsilon_{2}\left(\sigma_{0}, H\right)\left(\zeta\left(2 \sigma_{0}\right)+\epsilon_{1}\left(\sigma_{0}, H\right)\right)}, \\
\mathcal{E}_{1}= & \epsilon_{1}+\epsilon_{2}+\epsilon_{3} .
\end{aligned}
$$

Then, for all $T \geq H_{0}$, we have

$$
\begin{gathered}
\frac{1}{T-H} \int_{H}^{T}\left|\zeta\left(\sigma_{0}+i t\right)\right|^{2} d t \leq \zeta\left(2 \sigma_{0}\right)+\mathcal{E}_{1}\left(\sigma_{0}, H\right), \\
\text { and } \int_{H}^{T} \log \left|\zeta\left(\sigma_{0}+i t\right)\right| d t \leq \frac{T-H}{2} \log \left(\zeta\left(2 \sigma_{0}\right)+\mathcal{E}_{1}\left(\sigma_{0}, H\right)\right) .
\end{gathered}
$$

For the rest of this article $H, T, \sigma_{0}$, and $\sigma$ satisfy

$H_{0}=3.061 \cdot 10^{10}, 10^{3} \leq H \leq H_{0} \leq T, 0.5208<\sigma_{0}<0.9723, \sigma_{1}=1.5002, \sigma_{0}<\sigma<\sigma_{1}$.

\section{Approximate formula for $\zeta(\sigma+i t)$ - Proof of Theorem 1.2}

Let $s=\sigma+i t$ with $1 / 2<\sigma<1$ and $t \geq 2$. Let $x=c t$ with $c>\frac{1}{2 \pi}$, and let $N$ be a positive integer. Theorem 1.2 gives an explicit version of an approximation formula for zeta, as proven by Hardy and Littlewood in [12].

Proof. We start with the classical identity [31, equation 3.5.3]

$$
\zeta(s)-\sum_{1 \leq n<x} \frac{1}{n^{s}}=\sum_{x \leq n \leq N} \frac{1}{n^{s}}+s \int_{N}^{\infty} \frac{((u))}{u^{s+1}} d u-\frac{N^{1-s}}{1-s}-\frac{1}{2} N^{-s},
$$


where $((u))=[u]-u+1 / 2$. The summation formula [31, equation 2.1.2] gives

$$
\sum_{x \leq n<N} \frac{1}{n^{s}}=\int_{x}^{N} \frac{d u}{u^{s}}-\frac{((x))}{x^{s}}+s \int_{x}^{N} \frac{((u))}{u^{1+s}} d u=\frac{N^{1-s}-x^{1-s}}{1-s}-\frac{((x))}{x^{s}}+s \int_{x}^{N} \frac{((u))}{u^{1+s}} d u .
$$

We have the bounds

$$
\left|\frac{x^{1-s}}{1-s}\right| \leq \frac{x^{1-\sigma}}{t},\left|\frac{((x))}{x^{s}}\right| \leq \frac{x^{-\sigma}}{2},\left|s \int_{N}^{\infty} \frac{((u))}{u^{s+1}} d u\right| \leq \frac{|s|}{2} \int_{N}^{\infty} \frac{1}{u^{\sigma+1}} d u=\frac{|s|}{2 \sigma N^{\sigma}} .
$$

Thus

$$
\left|\zeta(s)-\sum_{1 \leq n<x} \frac{1}{n^{s}}\right| \leq x^{1-\sigma} t^{-1}+\frac{x^{-\sigma}}{2}+\left|s \int_{x}^{N} \frac{((u))}{u^{1+s}} d u\right|+\frac{|s|}{2 \sigma} N^{-\sigma}+\frac{1}{2} N^{-\sigma} .
$$

The choice $x=c t$ is made to balance the error term $x^{1-\sigma} t^{-1}+\frac{x^{-\sigma}}{2}$. We appeal to the Fourier series of $((x))$ to obtain a smaller bound for the integral expression. For $u \notin \mathbb{N}$, we have [31, p. 74]

$$
((u))=[u]-u+1 / 2=\frac{1}{\pi} \sum_{\nu=1}^{\infty} \frac{\sin (2 \pi \nu u)}{\nu} .
$$

Lebesgue's bounded convergence theorem applies, and we can exchange the order of the integral and the summation. We obtain

$$
\int_{x}^{N} \frac{((u))}{u^{1+s}} d u=\frac{1}{\pi} \sum_{\nu=1}^{\infty} \frac{1}{\nu} \int_{x}^{N} \frac{\sin (2 \pi \nu u)}{u^{1+s}} d u=\sum_{\nu=1}^{\infty} \frac{I(\nu)-I(-\nu)}{\nu},
$$

where the integral $I$ is given by

$$
I(h)=\frac{1}{2 \pi i} \int_{x}^{N} \frac{e^{2 i \pi\left(h u-\frac{t \log u}{2 \pi}\right)}}{u^{\sigma+1}} d u=\frac{1}{2 \pi} \int_{x}^{N} F(h, u) d\left(e^{2 \pi i(f(u)+h u)}\right)
$$

with $F(h, u)=\frac{u^{-\sigma}}{t-2 \pi u h}$ and $f(u)=-\frac{t \log u}{2 \pi}$. Since

$$
\frac{\partial}{\partial u} F(h, u)=u^{-\sigma} \frac{-\sigma t u^{-1}+2 \pi h(\sigma+1)}{(t-2 \pi u h)^{2}},
$$

it is easy to check that $F(-\nu, u)$ is positive and decreases with $u$, and that $F(\nu, u)$ is negative and increases with $u$.

We now apply the second mean value theorem from [32, section 12.3]:

Lemma 2.1. If $j(x)$ is integrable over $(a, b)$, and $\phi(x)$ is positive, bounded, and non-increasing, then there exists $\xi \in(a, b)$ such that

$$
\int_{a}^{b} \phi(x) j(x) d x=\phi(a+0) \int_{a}^{\xi} j(x) d x .
$$

First, we consider $I(-\nu)$. We separate the real and imaginary part in $d\left(e^{2 \pi i(f(u)+h u)}\right)$ in (2.4) and we apply the Lemma for $\phi(u)=F(-\nu, u)$. We 
consider $j(u) d u=d(\cos (2 \pi(f(u)-\nu u)))$, and $j(u) d u=d(\sin (2 \pi(f(u)-$ $\nu u))$ ) respectively. We obtain that there exist $\xi_{1}, \xi_{2} \in(x, N)$ such that

$$
\begin{aligned}
2 \pi I(-\nu)=F(-\nu, x) \cos \left(2 \pi\left(f\left(\xi_{1}\right)-\nu \xi_{1}\right)\right)-F(-\nu, x) e^{2 \pi i(f(x)-\nu x)} & \\
& +i F(-\nu, x) \sin \left(2 \pi\left(f\left(\xi_{2}\right)-\nu \xi_{2}\right)\right) .
\end{aligned}
$$

It follows that

$$
|I(-\nu)| \leq \frac{3}{2 \pi} F(-\nu, x)=\frac{3}{2 \pi} \frac{(c t)^{-\sigma}}{t+2 \pi c t \nu} \leq \frac{3}{(2 \pi)^{2}} \frac{c^{-\sigma-1} t^{-\sigma-1}}{\nu} .
$$

A similar argument applies to $I(\nu)$. We obtain

$$
|I(\nu)| \leq-\frac{3}{2 \pi} F(\nu, c t)=\frac{3}{2 \pi} \frac{(c t)^{-\sigma}}{2 \pi c t \nu-t} \leq\left\{\begin{array}{l}
\frac{3}{2 \pi} \frac{c^{-\sigma} t^{-\sigma-1}}{\nu-1} \text { if } \nu \geq 2 \\
\frac{3}{2 \pi} \frac{c^{-\sigma} t^{-\sigma-1}}{2 \pi c-1} \text { if } \nu=1
\end{array}\right.
$$

Using the simplification $\sum_{\nu=2}^{\infty} \frac{1}{\nu(\nu-1)}=1, \sum_{\nu=1}^{\infty} \frac{1}{\nu^{2}}=\zeta(2)$, and $\frac{|s|}{t} \leq \sqrt{1+1 / t^{2}}$, we put together (2.3),$(2.5)$, and (2.6), and obtain the bound

$$
\begin{aligned}
\left|s \int_{x}^{N} \frac{((u))}{u^{1+s}} d u\right| \leq|s| \sum_{\nu=1}^{\infty} \frac{|I(\nu)|+|I(-\nu)|}{\nu} & \\
& \leq \frac{3 \sqrt{1+1 / t^{2}}}{2 \pi}\left(1+\frac{1}{2 \pi c-1}+\frac{\zeta(2)}{2 \pi c}\right) c^{-\sigma} t^{-\sigma} .
\end{aligned}
$$

Letting $N \rightarrow \infty$, inequality (2.2) becomes

$$
\left|\zeta(s)-\sum_{1 \leq n<c t} \frac{1}{n^{s}}\right| \leq\left(c+\frac{1}{2}+\frac{3 \sqrt{1+1 / t^{2}}}{2 \pi}\left(1+\frac{1}{2 \pi c-1}+\frac{\zeta(2)}{2 \pi c}\right)\right)(c t)^{-\sigma} .
$$

Remark 2.2. A careful reading of Cheng's proof shows that his error term has size $\mathcal{O}\left(t^{1-2 \sigma}\right)$, instead of our $\mathcal{O}\left(t^{-\sigma}\right)$. This comes from he fact that he bounds directly the terms $\frac{N^{1-s}}{1-s}$, instead of eliminating them as we did.

\section{EXPLiCIT UPPER BOUND FOR THE SECOND MOMENT OF ZETA -}

\section{Proof of TheOREM 1.4}

We recall that $\sigma_{0}, T, H$ are as in (1.11). By Theorem 1.2, we have the identity

$$
\frac{1}{T-H} \int_{H}^{T}\left|\zeta\left(\sigma_{0}+i t\right)\right|^{2} d t=D\left(\sigma_{0}, T, H\right)+E_{1}\left(\sigma_{0}, T, H\right)+E_{2}\left(\sigma_{0}, T, H\right)+E_{3}\left(\sigma_{0}, T, H\right)
$$


where

$$
\begin{aligned}
& D\left(\sigma_{0}, T, H\right)=\frac{1}{T-H} \int_{H}^{T} \sum_{1 \leq n<t} \frac{1}{n^{2 \sigma_{0}}} d t \\
& E_{1}\left(\sigma_{0}, T, H\right)=\frac{2}{T-H} \int_{H}^{T} \sum_{1 \leq n<m<t} \frac{\cos (t \log (m / n))}{(n m)^{\sigma_{0}}} d t \\
& E_{2}\left(\sigma_{0}, T, H\right)=\frac{1}{T-H} \int_{H}^{T}\left|R\left(\sigma_{0}+i t\right)\right|^{2} d t \\
& E_{3}\left(\sigma_{0}, T, H\right)=\frac{2}{T-H} \mathfrak{R e} \int_{H}^{T} \sum_{1 \leq n<t} \frac{R\left(\sigma_{0}+i t\right)}{n^{\sigma_{0}+i t}} d t .
\end{aligned}
$$

We recall here some basic inequalities that we use throughout the following argument. Let $A, B \in \mathbb{N}$. If $f$ is decreasing and positive, then

$$
\sum_{A \leq j \leq B} f(j) \leq f(A)+\int_{A}^{B} f(u) d u
$$

For $\sigma_{0}>1 / 2$, we bound trivially the diagonal term:

$$
D\left(\sigma_{0}, T, H\right) \leq \zeta\left(2 \sigma_{0}\right)
$$

We interchange summation order in the off-diagonal terms $E_{1}\left(\sigma_{0}, T, H\right)$ and use the fact that $\int_{u}^{v} \cos (a t) d t \leq \frac{2}{a}$ when $a \neq 0$ :

$$
E_{1}\left(\sigma_{0}, T, H\right) \leq \frac{4}{T-H} \sum_{1 \leq n<m<T} \frac{(n m)^{-\sigma_{0}}}{\log (m / n)}
$$

We use the fact that, for $\lambda>1$ and $\sigma<1, \frac{1}{\log \lambda} \leq 1+\frac{\lambda^{1-\sigma}}{\lambda-1}$. Taking $\lambda=\frac{m}{n}$, we obtain

$$
E_{1}\left(\sigma_{0}, T, H\right) \leq \frac{4}{T-H} \sum_{1 \leq n<m<T}(n m)^{-\sigma_{0}}+\frac{4}{T-H} \sum_{1 \leq n<m<T} \frac{m^{1-2 \sigma_{0}}}{m-n} .
$$

For the first sum, we complete the square

$\sum_{1 \leq n<m<T}(n m)^{-\sigma_{0}}=\frac{1}{2}\left(\sum_{k<T} k^{-\sigma_{0}}\right)^{2}-\frac{1}{2} \sum_{k<T} k^{-2 \sigma_{0}}=\frac{1}{2}\left(\sum_{k<T} k^{-\sigma_{0}}\right)^{2}-\frac{1}{2}\left(\zeta\left(2 \sigma_{0}\right)-\sum_{k \geq T} k^{-2 \sigma_{0}}\right)$,

and use (3.2) with $f(t)=t^{-\sigma_{0}}$ and $f(t)=t^{-2 \sigma_{0}}$ to bound the resulting sums. We obtain

$$
\sum_{1 \leq n<m<T}(n m)^{-\sigma_{0}} \leq \frac{T^{2\left(1-\sigma_{0}\right)}}{2\left(1-\sigma_{0}\right)^{2}}-\frac{\sigma_{0} T^{1-\sigma_{0}}}{\left(1-\sigma_{0}\right)^{2}}+\frac{\sigma_{0}^{2}}{2\left(1-\sigma_{0}\right)^{2}}-\frac{1}{2} \zeta\left(2 \sigma_{0}\right)-\frac{T^{1-2 \sigma_{0}}}{2\left(1-2 \sigma_{0}\right)}+\frac{T^{-2 \sigma_{0}}}{2} .
$$

We consider $k=m-n$ and separate variables in the second sum of (3.4) and use (3.2), with $f(t)=t^{1-2 \sigma_{0}}$ and $f(t)=t^{-1}$, to bound the resulting 
sums:

$$
\begin{aligned}
& \sum_{1 \leq n<m<T} \frac{m^{1-2 \sigma_{0}}}{m-n} \leq\left(\sum_{1 \leq m<T} m^{1-2 \sigma_{0}}\right)\left(\sum_{1 \leq k<T} k^{-1}\right) \\
\leq & \frac{(\log T) T^{2\left(1-\sigma_{0}\right)}}{2\left(1-\sigma_{0}\right)}+\frac{T^{2\left(1-\sigma_{0}\right)}}{2\left(1-\sigma_{0}\right)}+\log T+1-\frac{1}{2\left(1-\sigma_{0}\right)}-\frac{\log T}{2\left(1-\sigma_{0}\right)} .
\end{aligned}
$$

Together with (3.4), (3.5) and (3.6), we obtain

$$
\begin{aligned}
E_{1}\left(\sigma_{0}, T, H\right) & \leq \frac{4 T}{T-H}\left(\frac{(\log T) T^{1-2 \sigma_{0}}}{2\left(1-\sigma_{0}\right)}-\frac{2 \sigma_{0}-1}{2\left(1-\sigma_{0}\right)} \frac{\log T}{T}+\left(\frac{1-3 \sigma_{0}+3 \sigma_{0}^{2}}{2\left(1-\sigma_{0}\right)^{2}}-\frac{1}{2} \zeta\left(2 \sigma_{0}\right)\right) \frac{1}{T}\right. \\
& \left.+\frac{2-\sigma_{0}}{2\left(1-\sigma_{0}\right)^{2}} T^{1-2 \sigma_{0}}-\frac{\sigma_{0} T^{-\sigma_{0}}}{\left(1-\sigma_{0}\right)^{2}}+\frac{T^{-2 \sigma_{0}}}{2\left(2 \sigma_{0}-1\right)}+\frac{1}{2} T^{-2 \sigma_{0}-1}\right) .
\end{aligned}
$$

We denote

$$
\begin{aligned}
& E_{11}\left(\sigma_{0}, T\right)=\frac{(\log T) T^{1-2 \sigma_{0}}}{2\left(1-\sigma_{0}\right)}-\frac{2 \sigma_{0}-1}{2\left(1-\sigma_{0}\right)} \frac{\log T}{T}, \\
& E_{12}\left(\sigma_{0}, T\right)=\left(\frac{1-3 \sigma_{0}+3 \sigma_{0}^{2}}{2\left(1-\sigma_{0}\right)^{2}}-\frac{1}{2} \zeta\left(2 \sigma_{0}\right)\right) \frac{1}{T} \\
& E_{13}\left(\sigma_{0}, T\right)=\frac{2-\sigma_{0}}{2\left(1-\sigma_{0}\right)^{2}} T^{1-2 \sigma_{0}}-\frac{\sigma_{0} T^{-\sigma_{0}}}{\left(1-\sigma_{0}\right)^{2}}, \\
& E_{14}\left(\sigma_{0}, T\right)=\frac{T^{-2 \sigma_{0}}}{2\left(2 \sigma_{0}-1\right)}+\frac{1}{2} T^{-2 \sigma_{0}-1}
\end{aligned}
$$

and we now study their behavior with respect to $T \geq H_{0}$. It is immediate that $E_{14}$ decreases with $T$. Considering the fact that $\frac{1-3 \sigma_{0}+3 \sigma_{0}^{2}}{2\left(1-\sigma_{0}\right)^{2}}-\frac{1}{2} \zeta\left(2 \sigma_{0}\right)$ changes sign at $\sigma_{0}=0.679785 \ldots$, we obtain

$$
E_{12}\left(\sigma_{0}, T\right) \leq \max \left(0, E_{12}\left(\sigma_{0}, H_{0}\right)\right) .
$$

For $0.5208<\sigma_{0}<1$, we find

$$
\frac{\partial E_{11}\left(\sigma_{0}, T\right)}{\partial T}=\frac{-\left(T^{2\left(1-\sigma_{0}\right)}-1\right)\left(\left(2 \sigma_{0}-1\right)(\log T)-1\right)+2\left(1-\sigma_{0}\right)}{2\left(1-\sigma_{0}\right) T^{2}} \leq 0,
$$

and, when $\sigma_{0} \leq 0.9723$, that

$$
\frac{\partial E_{13}\left(\sigma_{0}, T\right)}{\partial T}=\left(-\frac{\left(2-\sigma_{0}\right)\left(2 \sigma_{0}-1\right)}{2} T^{1-\sigma_{0}}+\sigma_{0}^{2}\right) \frac{T^{-1-\sigma_{0}}}{\left(1-\sigma_{0}\right)^{2}} \leq 0 .
$$

Thus $E_{11}\left(\sigma_{0}, T\right)$ and $E_{13}\left(\sigma_{0}, T\right)$ decrease with $T \geq H_{0}$. We conclude that, for $T \geq H_{0}$ and $0.5208 \leq \sigma_{0} \leq 0.9723$,

$$
E_{1}\left(\sigma_{0}, T, H\right) \leq \frac{4 H_{0}}{H_{0}-H}\left(\frac{\left(\log H_{0}\right) H_{0}^{1-2 \sigma_{0}}}{2\left(1-\sigma_{0}\right)}-\frac{2 \sigma_{0}-1}{2\left(1-\sigma_{0}\right)} \frac{\log H_{0}}{H_{0}}\right.
$$

$$
\left.+\frac{\max \left(0, \frac{1-3 \sigma_{0}+3 \sigma_{0}^{2}}{2\left(1-\sigma_{0}\right)^{2}}-\frac{\zeta\left(2 \sigma_{0}\right)}{2}\right)}{H_{0}}+\frac{\left(2-\sigma_{0}\right) H_{0}^{1-2 \sigma_{0}}}{2\left(1-\sigma_{0}\right)^{2}}-\frac{\sigma_{0} H_{0}^{-\sigma_{0}}}{\left(1-\sigma_{0}\right)^{2}}+\frac{H_{0}^{-2 \sigma_{0}}}{2\left(2 \sigma_{0}-1\right)}+\frac{H_{0}^{-2 \sigma_{0}-1}}{2}\right) .
$$


Theorem 1.2 gives

$$
E_{2}\left(\sigma_{0}, T, H\right) \leq c_{0}^{2} \frac{1}{T-H} \int_{H}^{T} t^{-2 \sigma_{0}} d t \leq \frac{c_{0}^{2}}{2 \sigma_{0}-1} \frac{H^{-\left(2 \sigma_{0}-1\right)}-H_{0}^{-\left(2 \sigma_{0}-1\right)}}{H_{0}-H} .
$$

We use the Cauchy-Schwarz inequality to bound $E_{3}$ :

$$
\begin{aligned}
E_{3}\left(\sigma_{0}, T, H\right) & \leq 2\left(\frac{1}{T-H} \int_{H}^{T}|\mathfrak{R e} R(s)|^{2} d t\right)^{\frac{1}{2}}\left(\frac{1}{T-H} \int_{H}^{T}\left|\sum_{1 \leq n<t} \frac{1}{n^{\sigma_{0}+i t}}\right|^{2} d t\right)^{\frac{1}{2}} \\
& \leq 2 \sqrt{E_{2}\left(\sigma_{0}, T, H\right)\left(D\left(\sigma_{0}, T, H\right)+E_{1}\left(\sigma_{0}, T, H\right)\right)} \\
& \leq 2 \sqrt{\epsilon_{2}\left(\sigma_{0}, H\right)\left(\zeta\left(2 \sigma_{0}\right)+\epsilon_{1}\left(\sigma_{0}, H\right)\right)} .
\end{aligned}
$$

The definitions of $\epsilon_{1}, \epsilon_{2}, \epsilon_{3}$ follow from (3.7), (3.8), and (3.9). The proof is achieved by putting together (3.1), (3.3), (3.7), (3.8), (3.9), and by applying the following bound for concave functions

$$
\int_{H}^{T} \log \left|\zeta\left(\sigma_{0}+i t\right)\right| d t \leq \frac{T-H}{2} \log \left(\frac{1}{T-H} \int_{H}^{T}\left|\zeta\left(\sigma_{0}+i t\right)\right|^{2} d t\right) .
$$

4. A LOWER BOUND FOR $\log |\zeta(s)|$ WHEN $\sigma>1$.

Lemma 4.1. Let $2 \leq H \leq T$ and $\sigma_{1}=1.5002$. Then

$$
\int_{H}^{T} \log \left|\zeta\left(\sigma_{1}+i t\right)\right| d t \geq-\mathcal{E}_{2}, \text { with } \mathcal{E}_{2}=1.7655 \text {. }
$$

Proof. Let $s=\sigma_{1}+i t$. It follows from the Euler product that

$$
\log |\zeta(s)|=\mathfrak{R e} \sum_{n \geq 2} \frac{\Lambda(n)}{(\log n) n^{s}} .
$$

Thus

$$
\int_{H}^{T} \log \left|\zeta\left(\sigma_{1}+i t\right)\right| d t=\sum_{n \geq 2} \frac{\Lambda(n)(\sin (T \log n)-\sin (H \log n))}{(\log n)^{2} n^{\sigma_{1}}} \geq-2 \sum_{n \geq 2} \frac{\Lambda(n)}{(\log n)^{2} n^{\sigma_{1}}} .
$$

We truncate the sum at $N_{0}=10^{3}$ and bound the tail

$$
\sum_{n>N_{0}} \frac{\Lambda(n)}{(\log n)^{2} n^{\sigma_{1}}} \leq \frac{1}{\left(\log N_{0}\right)^{2}}\left(-\frac{\zeta^{\prime}}{\zeta}\left(\sigma_{1}\right)-\sum_{n \leq N_{0}} \frac{\Lambda(n)}{n^{\sigma_{1}}}\right) .
$$

We obtain

$\int_{H}^{T} \log \left|\zeta\left(\sigma_{1}+i t\right)\right| d t \geq-2\left(\frac{-\frac{\zeta^{\prime}}{\zeta}\left(\sigma_{1}\right)}{\left(\log N_{0}\right)^{2}}+\sum_{n \leq N_{0}} \frac{\Lambda(n)}{n^{\sigma_{1}}}\left(\frac{1}{(\log n)^{2}}-\frac{1}{\left(\log N_{0}\right)^{2}}\right)\right)$,

and a numerical calculation with Maple gives the value for the above left term. 
5. ExPLICIT BOUNDS FOR $\int_{\sigma_{0}}^{\sigma_{1}} \arg \zeta(\tau+i T) d \tau$.

Lemma 5.1. Let $\eta=0.0001, \sigma_{1}=3 / 2+2 \eta=1.5002$. Let $\sigma_{0}, T, H$ satisfy $\sigma_{0}<\sigma_{1}, 2 \leq H \leq T$. Then

$\int_{\sigma_{0}}^{\sigma_{1}} \arg \zeta(\tau+i T) d \tau-\int_{\sigma_{0}}^{\sigma_{1}} \arg \zeta(\tau+i H) d \tau \leq \mathcal{E}_{3}\left(\sigma_{0}\right) \log (H T)+\mathcal{E}_{4}\left(\sigma_{0}, H\right)$

with

$\mathcal{E}_{3}\left(\sigma_{0}\right)=\frac{\pi(1+2 \eta)\left(\sigma_{1}-\sigma_{0}\right)}{4 \log 2}$

$\mathcal{E}_{4}\left(\sigma_{0}, H\right)=\frac{\pi\left(\sigma_{1}-\sigma_{0}\right)}{\log 2} \log \left(3 \frac{H+3(1+\eta)}{H-(1+2 \eta)}\left(\frac{3(1+\eta) / H+1}{2 \pi}\right)^{\frac{1+2 \eta}{2}} \frac{\zeta(1+\eta)^{4}}{\zeta(2(1+\eta))^{2}}\right)$.

It suffices to bound an integral of the form

$$
\int_{\sigma_{0}}^{\sigma_{1}} \arg \zeta(\tau+i t) d \tau
$$

with $t \geq H$. We only make use of the convexity bound for $\zeta(s)$.

Proof. Let $\omega \in \mathbb{C}$ and $N \in \mathbb{N}$. Following Rosser's modification of Backlund's

trick ([1, equation (32)] and [24, page 223]), we introduce $f_{t}(\omega)=\frac{1}{2}\left(\zeta(\omega+i t)^{N}+\zeta(\omega-i t)^{N}\right)$.

We denote $n$ to be the number of real zeros of $f_{t}(\tau)=\mathfrak{R e} \zeta(\tau+i t)^{N}$ in the interval $\sigma_{0}<\tau<\sigma_{1}$. The interval is split into $n+1$ subintervals and on each of them $\arg \zeta(\tau+i t)^{N}$ changes by at most $\pi$. Thus

$$
\left|\int_{\sigma_{0}}^{\sigma_{1}} \arg \zeta(\tau+i t) d \tau\right|=\frac{1}{N}\left|\int_{\sigma_{0}}^{\sigma_{1}} \arg \zeta(\tau+i t)^{N} d \tau\right| \leq \frac{\left(\sigma_{1}-\sigma_{0}\right)(n+1) \pi}{N} .
$$

We denote $n(r)$ the number of zeros of $f_{t}$ in the circle centered at $1+\eta+i t$, and with radius $r$. For $r \geq 1 / 2+\eta$, the segment $\left[\sigma_{0}, \sigma_{1}\right]$ is contained in $[1+\eta-r, 1+\eta+r]$, thus $n \leq n(r)$. The following version of Jensen's formula [29, p. 137, equation (2)],

$$
\log \left|f_{t}(1+\eta)\right|+\int_{0}^{1+2 \eta} \frac{n(r)}{r} d r=\frac{1}{2 \pi} \int_{-\pi / 2}^{3 \pi / 2} \log \left|f_{t}\left(1+\eta+(1+2 \eta) e^{i \theta}\right)\right| d \theta
$$

allows us to deduce an upper bound for $n$ :

$n \leq \frac{1}{\log 2} \int_{0}^{1+2 \eta} \frac{n(r)}{r} d r \leq \frac{1}{2 \pi \log 2} \int_{-\pi / 2}^{3 \pi / 2} \log \left|f_{t}\left(1+\eta+(1+2 \eta) e^{i \theta}\right)\right| d \theta-\frac{\log \left|f_{t}(1+\eta)\right|}{\log 2}$

We write $\zeta(1+\eta+i t)=R e^{i \phi}$. Thus $f_{t}(1+\eta)=\mathfrak{R e}\left(\zeta(1+\eta+i t)^{N}\right)=$ $R^{N} \cos (N \phi)$. We choose a sequence of $N$ 's such that $\lim _{N \rightarrow \infty} N \phi=0(\bmod 2 \pi)$. 
Thus

$$
\begin{aligned}
\log \left|f_{t}(1+\eta)\right|=N \log ((1+o(1)) R)= & N \log ((1+o(1))|\zeta(1+\eta+i t)|) \\
& \geq N \log \left(\frac{\zeta(2(1+\eta))}{\zeta(1+\eta)}\right)+o_{N}(1),
\end{aligned}
$$

where $o_{N}(1) \rightarrow 0$ when $N \rightarrow \infty$. We now split the integral in the left term of inequality (5.4) depending on the sign of $\cos \theta$. For $\theta \in(-\pi / 2, \pi / 2)$, $\mathfrak{R e}\left(1+\eta+(1+2 \eta) e^{i \theta} \pm i t\right)>1+\eta>1$, and we use the trivial bound

$$
\left|\zeta\left(1+\eta+(1+2 \eta) e^{i \theta} \pm i t\right)\right| \leq \zeta(1+\eta),
$$

giving

$$
\int_{-\pi / 2}^{\pi / 2} \log \left|f_{t}\left(1+\eta+(1+2 \eta) e^{i \theta}\right)\right| d \theta \leq N \pi \log (\zeta(1+\eta)) .
$$

For $\theta \in(\pi / 2,3 \pi / 2)$, we use Rademacher's bound [17, equation (7.4)]:

$$
|\zeta(s)| \leq 3 \frac{|1+s|}{|1-s|}\left(\frac{|1+s|}{2 \pi}\right)^{\frac{1+\eta-\Re e s}{2}} \zeta(1+\eta)
$$

with $s=1+\eta+(1+2 \eta) e^{i \theta} \pm i t$. Since

$|1+s| \leq t+3(1+\eta),|1-s| \geq|\mathfrak{I m} s| \geq t-(1+2 \eta)$, and $0 \leq 1+\eta-\mathfrak{R e} s \leq 1+2 \eta$

then

$$
\begin{aligned}
\int_{\pi / 2}^{3 \pi / 2} \log \mid f_{t} & \left(1+\eta+(1+2 \eta) e^{i \theta}\right) \mid d \theta \\
& \leq N \pi \log \left(3 \frac{t+3(1+\eta)}{t-(1+2 \eta)}\left(\frac{t+3(1+\eta)}{2 \pi}\right)^{\frac{1+2 \eta}{2}} \zeta(1+\eta)\right) .
\end{aligned}
$$

Together with (5.4), (5.5), (5.6), and (5.7), we deduce

$$
\begin{aligned}
& n \leq \frac{N}{2 \log 2} \log \left(3 \frac{t+3(1+\eta)}{t-(1+2 \eta)}\left(\frac{3(1+\eta)+t}{2 \pi}\right)^{\frac{1+2 \eta}{2}} \frac{\zeta(1+\eta)^{4}}{\zeta(2(1+\eta))^{2}}\right)+o_{N}(1) \\
\leq & \frac{N(1+2 \eta)}{4 \log 2} \log t+\frac{N}{2 \log 2} \log \left(3 \frac{t+3(1+\eta)}{t-(1+2 \eta)}\left(\frac{3(1+\eta) / t+1}{2 \pi}\right)^{\frac{1+2 \eta}{2}} \frac{\zeta(1+\eta)^{4}}{\zeta(2(1+\eta))^{2}}\right)+o_{N}(1) .
\end{aligned}
$$

Together with (5.3) and letting $N \rightarrow \infty$, we obtain

$$
\begin{aligned}
& \left|\int_{\sigma_{0}}^{\sigma_{1}} \arg \zeta(\tau+i t) d \tau\right| \leq \frac{\pi(1+2 \eta)\left(\sigma_{1}-\sigma_{0}\right)}{4 \log 2} \log t \\
& \quad+\frac{\pi\left(\sigma_{1}-\sigma_{0}\right)}{2 \log 2} \log \left(3 \frac{t+3(1+\eta)}{t-(1+2 \eta)}\left(\frac{3(1+\eta) / t+1}{2 \pi}\right)^{\frac{1+2 \eta}{2}} \frac{\zeta(1+\eta)^{4}}{\zeta(2(1+\eta))^{2}}\right) .
\end{aligned}
$$

Observing that the second term decreases with $t \geq H$ achieves the proof. 


\section{Explicit upper bounds For $N(\sigma, T)$ - Proof of Theorem 1.1}

Proof. We recall that $\sigma, \sigma_{0}, \sigma_{1}, H$ and $T$ satisfy (1.11). We consider the number $N(\sigma, T)$ of zeros $\varrho=\beta+i \gamma$ of zeta in the rectangle $\sigma<\beta<1$ and $H<\gamma<T$. Since $N(\sigma, H)=0$, we have

$$
N(\sigma, T) \leq \frac{1}{\sigma-\sigma_{0}} \int_{\sigma_{0}}^{\sigma_{1}}(N(\tau, T)-N(\tau, H)) d \tau .
$$

It follows from a lemma of Littlewood (see [31, (9.9.1)]) that

$$
\int_{\sigma_{0}}^{\sigma_{1}}(N(\tau, T)-N(\tau, H)) d \tau=-\frac{1}{2 \pi i} \int_{\mathcal{R}} \log \zeta(s) d s,
$$

where $\mathcal{R}$ is the rectangle with vertices $\sigma_{0}+i H, \sigma_{1}+i H, \sigma_{1}+i T$, and $\sigma_{0}+i T$.

Thus

$$
\begin{array}{r}
N(\sigma, T) \leq \frac{1}{2 \pi\left(\sigma-\sigma_{0}\right)}\left(\int_{H}^{T} \log \left|\zeta\left(\sigma_{0}+i t\right)\right| d t+\int_{\sigma_{0}}^{\sigma_{1}} \arg \zeta(\tau+i T) d \tau\right. \\
\left.-\int_{\sigma_{0}}^{\sigma_{1}} \arg \zeta(\tau+i H) d \tau-\int_{H}^{T} \log \left|\zeta\left(\sigma_{1}+i t\right)\right| d t\right) .
\end{array}
$$

We use Theorem 1.4. Lemma 4.1, and Lemma 5.1 respectively to bound these integrals:

$$
\begin{aligned}
& \int_{H}^{T} \log \left|\zeta\left(\sigma_{0}+i t\right)\right| d t \leq \frac{T-H}{2} \log \left(\zeta\left(2 \sigma_{0}\right)+\mathcal{E}_{1}\left(\sigma_{0}, H\right)\right), \\
& -\int_{H}^{T} \log \left|\zeta\left(\sigma_{1}+i t\right)\right| d t \leq \mathcal{E}_{2}, \\
& \int_{\sigma_{0}}^{\sigma_{1}} \arg \zeta(\tau+i T) d \tau-\int_{\sigma_{0}}^{\sigma_{1}} \arg \zeta(\tau+i H) d \tau \leq \mathcal{E}_{3}\left(\sigma_{0}\right) \log (H T)+\mathcal{E}_{4}\left(\sigma_{0}, H\right),
\end{aligned}
$$

where the $\mathcal{E}_{i}$ 's are defined respectively in (1.10), (4.1), (5.1), and (5.2). We obtain

$$
N(\sigma, T) \leq b_{1}\left(\sigma_{0}, H\right)(T-H)+b_{2}\left(\sigma_{0}, H\right) \log (T H)+b_{3}\left(\sigma_{0}, H\right),
$$

with

$b_{1}\left(\sigma_{0}, H\right)=\frac{\log \left(\zeta\left(2 \sigma_{0}\right)+\mathcal{E}_{1}\left(\sigma_{0}, H\right)\right)}{4 \pi\left(\sigma-\sigma_{0}\right)}, b_{2}\left(\sigma_{0}, H\right)=\frac{\mathcal{E}_{3}\left(\sigma_{0}\right)}{2 \pi\left(\sigma-\sigma_{0}\right)}, b_{3}\left(\sigma_{0}, H\right)=\frac{\mathcal{E}_{2}+\mathcal{E}_{4}\left(\sigma_{0}, H\right)}{2 \pi\left(\sigma-\sigma_{0}\right)}$.

It follows

$N(\sigma, T) \leq c_{1} T+c_{2} \log T+c_{3}$, with $c_{1}=b_{1}, c_{2}=b_{2}, c_{3}=-b_{1} H+b_{2} \log H+b_{3}$. 
Table 1 records values of the $b_{i}$ 's and $c_{i}$ 's computed for $H_{0}=3.061$. $10^{10}$. Specific choices of parameters $\sigma_{0}$ and $H$ are chosen in order to obtain good bounds for $N(\sigma, T)$ when $T$ is asymptotically large. The values of $\sigma_{0}, b_{1}, c_{1}, b_{2}$, and $c_{2}$ displayed in the table are rounded up to 4 decimal places. We take the ceiling of the values of $H, b_{3}$, and $c_{3}$.

TABLE 1. $N(\sigma, T) \leq b_{1}(T-H)+b_{2} \log (T H)+b_{3}$ and $N(\sigma, T) \leq c_{1} T+c_{2} \log T+c_{3}$, for $T \geq H_{0}$.

\begin{tabular}{|r|r|r|r|r|r|r|}
\hline$\sigma$ & $\sigma_{0}$ & $H$ & $b_{1}=c_{1}$ & $b_{2}=c_{2}$ & $b_{3}$ & $c_{3}$ \\
\hline 0.60 & 0.5229 & 19399 & 4.2288 & 2.2841 & 333 & -81673 \\
\hline 0.65 & 0.5552 & 40105 & 2.4361 & 1.7965 & 262 & -97414 \\
\hline 0.70 & 0.5873 & 91470 & 1.4934 & 1.4609 & 213 & -136370 \\
\hline 0.75 & 0.6096 & 169119 & 1.0031 & 1.1442 & 167 & -169449 \\
\hline 0.76 & 0.6136 & 188973 & 0.9355 & 1.0921 & 160 & -176604 \\
\hline 0.77 & 0.6175 & 210645 & 0.8750 & 1.0437 & 153 & -184134 \\
\hline 0.78 & 0.6213 & 234346 & 0.8205 & 0.9986 & 146 & -192120 \\
\hline 0.79 & 0.6250 & 260321 & 0.7714 & 0.9566 & 140 & -200644 \\
\hline 0.80 & 0.6287 & 288853 & 0.7269 & 0.9176 & 134 & -209795 \\
\hline 0.81 & 0.6324 & 320270 & 0.6864 & 0.8812 & 129 & -219667 \\
\hline 0.82 & 0.6361 & 354951 & 0.6495 & 0.8473 & 124 & -230367 \\
\hline 0.83 & 0.6398 & 393341 & 0.6156 & 0.8157 & 119 & -242009 \\
\hline 0.84 & 0.6435 & 435955 & 0.5846 & 0.7862 & 115 & -254724 \\
\hline 0.85 & 0.6472 & 483393 & 0.5561 & 0.7586 & 111 & -268658 \\
\hline 0.86 & 0.6510 & 536357 & 0.5297 & 0.7327 & 107 & -283978 \\
\hline 0.87 & 0.6548 & 595670 & 0.5053 & 0.7085 & 104 & -300872 \\
\hline 0.88 & 0.6587 & 662291 & 0.4827 & 0.6857 & 101 & -319555 \\
\hline 0.89 & 0.6626 & 737343 & 0.4617 & 0.6644 & 97 & -340272 \\
\hline 0.90 & 0.6667 & 822142 & 0.4421 & 0.6443 & 95 & -363301 \\
\hline 0.91 & 0.6708 & 918225 & 0.4238 & 0.6253 & 92 & -388959 \\
\hline 0.92 & 0.6750 & 1027390 & 0.4066 & 0.6075 & 89 & -417606 \\
\hline 0.93 & 0.6793 & 1151729 & 0.3905 & 0.5906 & 87 & -449647 \\
\hline 0.94 & 0.6838 & 1293683 & 0.3754 & 0.5747 & 84 & -485543 \\
\hline 0.95 & 0.6883 & 1456079 & 0.3612 & 0.5596 & 82 & -525807 \\
\hline 0.96 & 0.6930 & 1642194 & 0.3478 & 0.5452 & 80 & -571018 \\
\hline 0.97 & 0.6977 & 1855803 & 0.3352 & 0.5316 & 78 & -621815 \\
\hline 0.98 & 0.7026 & 2101249 & 0.3232 & 0.5187 & 76 & -678911 \\
\hline 0.99 & 0.7077 & 2383498 & 0.3118 & 0.5063 & 74 & -743087 \\
\hline & & & & & & \\
\hline
\end{tabular}


Aknowledgments. I would like to thank Olivier Ramaré for his comments on this article.

\section{REFERENCES}

[1] R. J. Backlund, Über die Nullstellen der Riemannschen Zetafunktion, Acta Mathematica, vol. 41 (1917), 345-375.

[2] Y. Cheng, An explicit upper bound for the Riemann Zeta-function near the line $\sigma=1$, Rocky Mountain J. Math. 29, Number 1 (1999), 115-140.

[3] Y. Cheng, Explicit estimate on primes between consecutive cubes, Rocky Mountain J. Math. 40, Number 1 (2010), 117-153.

[4] H. Daboussi and J. Rivat, Explicit upper bounds for exponential sums over primes, Math. Comp. 70 (2001), no. 233, 431-447

[5] P. Dusart, Autour de la fonction qui compte le nombre de nombres premiers Thése, Universit de Limoges, 1998.

[6] P. Dusart, Inégalités explicites pour $\psi(x), \theta(x), \pi(x)$ et les nombres premiers, C. R. Math. Acad. Sci. Soc. R. Can. 21 (1999), no. 2, 53-59.

[7] P. Dusart, The $k^{\text {th }}$ prime is greater than $k(\log k+\log \log k-1)$ for $k \geq 2$, Math. Comp. 68 (1999), no. 225, 411-415.

[8] P. Dusart, Estimates of some functions over primes without $R H$, arXiv preprint arXiv:1002.0442, 2010.

[9] L. Faber and H. Kadiri, New bounds for $\psi(x)$, preprint.

[10] K. Ford, Zero-free regions for the Riemann zeta function, Number theory for the millennium, II (Urbana, IL, 2000), 25-56

[11] X. Gourdon, The $10^{13}$ first zeros of the Riemann Zeta function, and zeros computation at very large height, available at http://numbers.computation.free.fr/Constants/Miscellaneous/zetazeros1e13-1e24.pdf

[12] G.H. Hardy \& J.E. Littlewood, The zeros of the Riemann zeta-function on the critical line, Math. Z. 10 (1921), 283-317.

[13] H. Helfgott, Minor arcs for Goldbach problem, preprint, arXiv:1205.5252.

[14] H. Kadiri Une région explicit sans zéros pour la fonctin $\zeta$ de Riemann, Acta Arith. 117 (2005), no. 4, 303-339.

[15] D. Platt, Computing degree 1 L-functions rigorously, Ph.D. Thesis, University of Bristol, 2011.

[16] D. Platt, Computing zeta on the half-line, preprint.

[17] H. Rademacher, On the Phragmén-Lindelöf theorem and some applications, Math. Z. 72 (1959), 192-204.

[18] O. Ramaré, On Snirelman's constant, Ann. Scuola Norm. Sup. Pisa Cl. Sci. 22 (1995), no. 4, 645-706.

[19] O. Ramaré, An explicit density estimate, preprint.

[20] O. Ramaré, Explicit estimates for the summatory function of $\Lambda(n) / n$ from the one of $\Lambda(n)$, to appear in Acta Arith.

[21] O. Ramaré, Explicit estimates on the summatory functions of the Moebius function with coprimality restrictions, preprint, available at http://math.univ-lille1.fr/ ramare/Maths/contenu.html.

[22] O. Ramaré, From explicit estimates for the primes to explicit estimates for the Moebius function, to appear in Acta Arith.

[23] O. Ramaré and R. Rumely, Primes in Arithmetic Progressions, Math. Comp. 65 (1996), no. 213, 397-425

[24] J.B. Rosser, Explicit bounds for some functions of prime numbers, Amer. J. Math. 63 (1941), 211-232.

[25] J.B. Rosser and L. Schoenfeld Approximate formulas for some functions of prime numbers, Illinois. J. Math. 6 (1962), 64-94. 
[26] J.B. Rosser and L. Schoenfeld Sharper bounds for Chebyshev functions $\psi(x)$ and $\theta(x)$, Math. Comp. 29 (1975), 243-269.

[27] J.B. Rosser and L. Schoenfeld Sharper bounds for Chebyshev functions $\psi(x)$ and $\theta(x)$ II, Math. Comp. 30 (1976), no. 134, 337-360.

[28] L. Schoenfeld, Sharper bounds for the Chebyshev functions $\psi$ and $\theta$ II, Math. Comp. 30 (1976), 337-360.

[29] E.M. Stein \& R. Shakarchi, Complex Analysis, Princeton Lectures in Analysis II, Princeton University Press.

[30] T. Tao, Every odd number greater than 1 is the sum of at most five primes, to appear in Math. Comp.

[31] E.C. Titchmarsh, The Theory of the Riemann Zeta-function, second edition, Oxford Science Publications.

[32] E.C. Titchmarsh, The Theory of Functions, second edition, Oxford University Press.

[33] T. Trudgian, An improved upper bound for the argument of the Riemann zetafunction on the critical line II, preprint, arXiv:1208.5846.

[34] J. van de Lune, H.J.J. te Riele, D.T. Winter On the zeros of the Riemann zetafunction in the critical strip. IV Math. Comp. 46 (1986), no. 174, 667-681.

[35] S. Wedeniwski - Zetagrid, Computational verification of the Riemann hypothesis Conference in Number Theory in Honour of Professor H.C. Williams, Alberta, Canada, May 2003.

http://www.zetagrid.net/zeta/math/zeta.result.100billion.zeros.html

Department of Mathematics and Computer Science, University of LethBridge, 4401 University Drive, Lethbridge, Alberta, T1K 3M4 Canada

E-mail address: habiba.kadiri@uleth.ca 